\title{
Benefits of Nonthermal Atmospheric Plasma Treatment on Dentin Adhesion
}

\author{
AP Ayres $\bullet$ PH Freitas $• \mathrm{~J}$ De Munck $\bullet$ A Vananroye $\bullet$ C Clasen \\ CT dos Santos Dias • M Giannini • B Van Meerbeek
}

\begin{abstract}
Clinical Relevance
After two years of water aging, the application of nonthermal atmospheric plasma onto dentin for $30 \mathrm{~s}$ showed higher microtensile bond strength of a multimode adhesive applied in etch-and-rinse mode. Plasma-treated dentin also resulted in higher nanohardness and Young's modulus of the hybrid layer in immediate evaluation and greater hydrophilicity.
\end{abstract}

\section{SUMMARY}

Objectives: This study aimed to evaluate the influence of two nonthermal atmospheric plasma (NTAP) application times and two storage times on the microtensile bond strength ( $\mu$ TBS) to dentin. The influence of NTAP on the mechanical properties of the dentin-resin interface was studied by analyzing nanohardness (NH) and Young's modulus (YM). Water contact angles of pretreated dentin and hydroxyapatite blocks were also measured to assess possible alterations in the surface hydrophilicity upon NTAP.

*Ana Paula Ayres, DDS, MSc, PhD, KU Leuven (University of Leuven), Department of Oral Health Sciences, BIOMAT \& UZ Leuven (University Hospitals Leuven), Dentistry, Leuven, Belgium

Pedro Henrique Freitas, DDS, MSc, PhD, Department of Restorative Dentistry, College of Dentistry, Faculty of Health Sciences, University of Manitoba, Winnipeg, MB, Canada

Jan De Munck, DDS, MSc, PhD. KU Leuven (University of Leuven), Department of Oral Health Sciences, BIOMAT \& UZ Leuven (University Hospitals Leuven), Dentistry, Leuven, Belgium

Anja Vananroye, MSc, PhD, Chemical Engineering Department, Soft Matter, Rheology and Technology, KU Leuven, University of Leuven, Leuven, Belgium.

Christian Clasen, MSc, PhD, Chemical Engineering Depart-
Methods and Materials: Forty-eight human molars were used in a split-tooth design $(n=8)$. Midcoronal exposed dentin was flattened by a 600-grit SiC paper. One-half of each dentin surface received phosphoric acid conditioning, while the other half was covered with a metallic barrier and remained unetched. Afterward, NTAP was applied on the entire dentin surface (etched or not) for 10 or 30 seconds. The control groups did not receive NTAP treatment. Scotchbond Universal (SBU; 3M ESPE) and a resin-based composite were applied to dentin following the manufacturer's

ment, Soft Matter, Rheology and Technology, KU Leuven, University of Leuven, Leuven, Belgium

Carlos Tadeu dos Santos Dias, MSc, PhD, Exact Sciences Department, Luiz de Queiroz College of Agriculture, University of São Paulo, Piracicaba, SP, Brazil

Marcelo Giannini, DDS, MSc, PhD, Department of Restorative Dentistry, Piracicaba Dental School, State University of Campinas, Piracicaba, SP, Brazil

Bart Van Meerbeek, DDS, MSc, PhD, KU Leuven (University of Leuven), Department of Oral Health Sciences, BIOMAT \& UZ Leuven (University Hospitals Leuven), Dentistry, Leuven, Belgium

*Corresponding author: Av. Nenê Sabino, 1801, UberabaMG 38055-500, Brazil; e-mail: anapaulaayres4@gmail.com DOI: $10.2341 / 17-123-\mathrm{L}$ 
instructions. After 24 hours of water storage at $37^{\circ} \mathrm{C}$, the specimens were sectioned perpendicular to the interface to obtain approximately six specimens or bonded beams (approximately $0.9 \mathrm{~mm}^{2}$ in cross-sectional area) representing the etch-and-rinse (ER) approach and another six specimens representing the selfetch (SE) approach. Half of the $\mu$ TBS specimens were immediately loaded until failure, while the other half were first stored in deionized water for two years. Three other bonded teeth were selected from each group $(n=3)$ for NH and YM evaluation. Water contact-angle analysis was conducted using a CAM200 (KSV Nima) goniometer. Droplet images of dentin and hydroxyapatite surfaces with or without 10 or 30 seconds of plasma treatment were captured at different water-deposition times $(5$ to 55 seconds).

Results: Two-way analysis of variance revealed significant differences in $\mu$ TBS of SBU to dentin after two years of water storage in the SE approach, without differences among treatments. After two years of water aging, the ER control and ER NTAP 10-second groups showed lower $\mu$ TBS means compared with the ER NTAP 30-second treated group. Nonthermal atmospheric plasma resulted in higher NH and YM for the hybrid layer. The influence of plasma treatment in hydrophilicity was more evident in the hydroxyapatite samples. Dentin hydrophilicity increased slightly after 10 seconds of NTAP, but the difference was higher when the plasma was used for 30 seconds.

Conclusions: Dentin NTAP treatment for 30 seconds contributed to higher $\mu$ TBS after two years of water storage in the ER approach, while no difference was observed among treatments in the SE evaluation. This result might be correlated to the increase in nanohardness and Young's modulus of the hybrid layer and to better adhesive infiltration, since dentin hydrophilicity was also improved. Although some effects were observed using NTAP for 10 seconds, the results suggest that 30 seconds is the most indicated treatment time.

\section{INTRODUCTION}

One of the most recent innovations in adhesive dentistry involves the treatment of different dental surfaces using nonthermal atmospheric plasma (NTAP), a novel technology that delivers highly reactive species in a gaseous medium at or below physiologic temperature. This technology tries to solve challenges commonly associated with hybridization of dentin during bonding procedures, therefore influencing the quality and longevity of the tooth-resin interface. A recently published review collected and summarized the current advances of NTAP in improving the durability of dentin bonding. ${ }^{1}$ The studies have demonstrated that NTAP applied on the dentin surface enhanced the bond strength of etch-and-rinse (ER) adhesives, ${ }^{2-4}$ but the results were more product dependent when employed to self-etch (SE) adhesives. ${ }^{5,6}$

Overall, NTAP has demonstrated efficacy in improving different properties of dental bonding because it provides higher wettability of the dentin surface,${ }^{2,6-9}$ improves resin polymerization, ${ }^{10,11}$ and allows deeper adhesive penetration. ${ }^{2,4,11}$ For the ER bonding technique, it was reported that a short plasma treatment could change the chemical structure of the exposed collagen fibrils and increase the hydrophilicity of the dentin surface, which allows better adhesive penetration into the dental collagen fibrils and enhances the dentin bond strength. ${ }^{11}$

A complex biomechanical entity is formed in the adhesive dental restoration that consists of the tooth substrate and the biomaterial. To predict the longterm performance of dental adhesives, it is necessary to understand their mechanical properties. The bonding area between the restorative resin composite and the dentinal cavity wall presents a gradual transition of different components, resulting in a heterogeneous gradient of physico-mechanical properties. ${ }^{12}$ Nevertheless, acid pretreatment modifies the hardness of the dentin surface, and the bonded interface zone might allow some flexibility with the hybridization process after resin polymerization. Such an elastic bonding area might have a strain capacity sufficient to relieve stresses between the composite shrinkage and the rigid dentin substrate, ${ }^{12,13}$ thereby preserving the integrity of marginal adaptation and consequently increasing the durability of the restoration.

The influence of the NTAP application on the mechanical properties of the adhesive-dentin interface is not established yet. The NTAP effect depends on treatment time, working gas, input power, pulse frequency of the plasma device, and also on other factors related to the substrate. ${ }^{1}$ Some investigations relate wettability enhancement associated with the NTAP treatment in different dental substrates. ${ }^{2,6-9}$ The plasma device of the present study (Surface Plasma Tool Model SAP; Surface-Engineering and Plasma Solution, Campinas, Brazil) improved the 
Table 1: Number of Specimens ( $n$ ) and Repetitions ( $r$ ) per Specimen Analyzed in Each of the Assays

\begin{tabular}{|c|c|c|c|c|c|c|c|}
\hline \multirow[t]{2}{*}{ Etching } & \multirow[t]{2}{*}{ NTAP } & \multicolumn{2}{|c|}{$\mu$ TBS } & \multirow[t]{2}{*}{ Nanohardness } & \multirow[t]{2}{*}{ Young's Modulus } & \multirow[t]{2}{*}{ Contact Angle: Dentin } & \multirow[t]{2}{*}{ Contact Angle: Hydroxyapatite } \\
\hline & & 1 -wk & $2-y$ & & & & \\
\hline \multirow[t]{4}{*}{ None } & & $n / r$ & $n / r$ & $n / r$ & $n / r$ & $n / r$ & $n / r$ \\
\hline & None & $8 / 6$ & $8 / 6$ & $3 / 5$ & $3 / 5$ & $5 / 1-3$ & $5 / 1-3$ \\
\hline & $10 \mathrm{~s}$ & $8 / 6$ & $8 / 6$ & $3 / 5$ & $3 / 5$ & $5 / 1-3$ & $5 / 1-3$ \\
\hline & $30 \mathrm{~s}$ & $8 / 6$ & $8 / 6$ & $3 / 5$ & $3 / 5$ & $5 / 1-3$ & $5 / 1-3$ \\
\hline \multirow[t]{3}{*}{ Phosphoric acid } & None & $8 / 6$ & $8 / 6$ & $3 / 5$ & $3 / 5$ & - & - \\
\hline & $10 \mathrm{~s}$ & $8 / 6$ & $8 / 6$ & $3 / 5$ & $3 / 5$ & - & - \\
\hline & $30 \mathrm{~s}$ & $8 / 6$ & $8 / 6$ & $3 / 5$ & $3 / 5$ & - & - \\
\hline
\end{tabular}

wettability of the zirconia surface, decreasing the contact angle (CA) by approximately $50 \% .{ }^{14}$ There is still a lack of information of the influence of this specific equipment and settings on the hydrophilicity of dentin tissue.

Therefore, this study aimed to assess the extent two application times of NTAP, 10 and 30 seconds, affect the long-term microtensile bond strength ( $\mu$ TBS) of one commercial multimode adhesive system. The influence of NTAP on the mechanical properties of the dentin-resin interface was studied by analyzing nanohardness, Young's modulus, and CA. The following null hypotheses were tested: 1 ) plasma treatment does not affect the dentin-bond strength tested immediately or after two years of aging, 2) NTAP treatment does not produce differences in the nanohardness and Young's modulus of the resin-dentin interface's structures (dentin, hybrid layer, and adhesive layer), and 3) the CA of the dentin and hydroxyapatite surfaces is not affected by the NTAP treatment.

\section{METHODS AND MATERIALS}

The distribution of specimens for each research subproject is detailed in Table 1. All statistical testing was performed at a preset alpha of 0.05 , and the values were calculated using SAS 9.3 Software (SAS Institute, Cary, NC, USA). Kolmogorov-Smirnov and Cramer-von Mises tests were used to verify normal distribution, and the Brown-Forsythe test was applied to homoscedasticity analysis.

\section{$\mu$ TBS}

Forty-eight noncarious human third molars (two groups per tooth; $n=8$ per experimental group) were stored in $0.5 \%$ chloramine/water immediately after extraction, then cleaned and stored in distilled water at $4^{\circ} \mathrm{C}$ to be used within three months. The occlusal third of the crowns was removed with a diamond saw, exposing the occlusal dentin surface (Isomet
100, Buehler, Lake Bluff, IL, USA). A standardized smear layer was produced under water irrigation using 600-grit SiC paper (Buehler-Met II, Buehler), and the flat surface was divided into two parts with similar areas using a thin diamond blade. One-half of each dentin surface was demineralized for 15 seconds with $34 \%$ phosphoric acid (Scotchbond Etchant, 3M ESPE, St Paul, MN, USA) in an ER approach, while the other half was covered with metallic paper and not etched in the SE approach. Afterward, NTAP was applied on the entire dentin surface (etched or not) for 10 or 30 seconds.

The plasma equipment used in this study (Surface Plasma Tool Model SAP, Surface-Engineering and Plasma Solution) consisted of a handheld unit using argon as the operating gas at a flow rate of $5.0 \mathrm{~L} / \mathrm{min}$. The plasma torch emerging at the exit nozzle was about $1.0 \mathrm{~mm}$ in diameter and was operated at room temperature $\left(22^{\circ} \mathrm{C}\right)$. A mobile base allowed keeping a distance of $10 \mathrm{~mm}$ between the nozzle and the dentin surface. Control groups did not receive NTAP treatment. Thus, the following groups were investigated $(n=8)$ :

1. SE control (unetched dentin, NTAP untreated)

2. SE NTAP 10 seconds

3. SE NTAP 30 seconds

4. ER control (etched dentin, NTAP untreated)

5. ER NTAP 10 seconds

6. ER NTAP 30 seconds

After the respective treatments, a multimode adhesive system (Scotchbond Universal, 3M ESPE; Table 2) was applied following the manufacturer's instructions and light-cured with a multiwavelength LED unit (VALO, Ultradent Products Inc, South Jordan, UT, USA) in standard mode with an output of about $1200 \mathrm{~mW} / \mathrm{cm}^{2}$, as measured by the MARC Patient Simulator (BlueLight Analytics, Halifax, NS, Canada). 
Table 2: Composition of Scotchbond Universal (3M ESPE) and Its Application Modes

\begin{tabular}{|c|c|c|}
\hline Composition (Batch No.) & Adhesive Mode & Application Method \\
\hline $\begin{array}{l}\text { Scotchbond Universal Etchant: } 34 \% \text { phosphoric acid, } \\
\text { water, synthetic amorphous silica, polyethylene glycol, } \\
\text { aluminum oxide (N489165) }\end{array}$ & $\begin{array}{l}\text { Two-step etch and } \\
\text { rinse (ER) }\end{array}$ & $\begin{array}{l}\text { Application of phosphoric acid gel etchant on dentin for } \\
15 \mathrm{~s} \text { and gentle air drying; rubbed application of the } \\
\text { adhesive for } 20 \mathrm{~s} \text {, followed by 5-s gentle air-drying and } \\
10 \text {-s light curing }\end{array}$ \\
\hline $\begin{array}{l}\text { Scotchbond Universal: 10-MDP, dimethacrylate resins, } \\
\text { HEMA, methacrylate-modified polyalkenoic acid } \\
\text { copolymer, filler, ethanol, water, initiators, and silane } \\
(621418), \mathrm{pH}=2.7\end{array}$ & $\begin{array}{l}\text { One-step self-etch } \\
\text { (SE) }\end{array}$ & $\begin{array}{l}\text { Rubbed application of the adhesive for } 20 \mathrm{~s} \text {, followed by } \\
5 \text {-s gentle air drying and 10-s light curing }\end{array}$ \\
\hline
\end{tabular}

A composite buildup (Filtek Supreme Ultra, shade A2 enamel, 3M ESPE) was made in layers (6-mm high), and each 2-mm layer was light-cured for 20 seconds with the VALO. The tooth root was removed $4 \mathrm{~mm}$ below the adhesive-dentin interface. After 24 hours of storage in deionized water $\left(37^{\circ} \mathrm{C}\right)$, the specimens were sectioned perpendicular to the interface into 0.9 -mm-thick bonded beams with a diamond saw under water cooling (Isomet, Buehler).

Approximately six specimens representing the ER approach and another six specimens representing the SE approach were obtained after sectioning the teeth. After one week, the beams were attached to a BIOMAT jig ${ }^{15}$ with cyanoacrylate glue (Model Repair II Blue; Dentsply-Sankin, Tochigiken, Japan) and tested in tension at a crosshead speed of $1 \mathrm{~mm} /$ min until failure in a universal testing machine (LRX, Lloyd, Hampshire, UK). The specimens from the other 24 teeth were stored in deionized water for two years. The storage medium was replaced every 15 days by deionized water at room temperature, and then the samples were kept at $37^{\circ} \mathrm{C}$ until the next water replacement.

A single failure stress value was calculated for each half of the tooth by averaging all beams obtained from that half tooth. The $\mu$ TBS $(\mathrm{MPa})$ was derived from dividing the force (Newton) applied at the time of fracture by the bonded area, of which the cross-sectional area was measured with a digital caliper (Starrett, Itu, SP, Brazil). The bond strength data $(\mathrm{MPa})$ was then analyzed by two-way analysis of variance (ANOVA) and Tukey's multiple comparison test for each adhesive technique (SE and ER).

After failure, the specimens were mounted on brass stubs and observed using a digital microscope system (Hirox KH-8700, Tokyo, Japan). The failure mode of each beam was classified into one of the following categories: cohesive failure in composite resin (C), cohesive failure in dentin (D), adhesive failure (A), or mixed failure of composite resin, adhesive, and dentin (M). Representative areas were photographed at $200 \times$ magnification. Data were submitted to chi-square analysis to demonstrate the effect of the factor "time" on failure mode.

\section{Nanohardness and Young's Modulus}

The same groups were used in this part of the study. Three bonded teeth from each group $(n=3)$ were longitudinally sectioned through the sample center with a diamond saw, under water cooling (Isomet, Buehler), to obtain two 1.5-mm-thick bonded slices. Each central slab was individually embedded in an epoxy resin (EpoxiCure, Buehler) and manually polished under water irrigation using $\mathrm{SiC}$ paper (Buehler-Met II, Buehler) with decreasing abrasiveness $(600,1000,1200,1500$, and 2000). Special soft discs (Apex Diamond Grinding Disks, Buehler) were associated with diamond polishing suspensions (MetaDi, Buehler) of 9-, 6-, 3-, 1-, and 0.5- $\mu \mathrm{m}$ grit size. Samples were ultrasonically cleaned with distilled water for five minutes between each polishing step.

The computer-controlled nano-indenter Hysitron Custom Triboindenter (Hysitron, Minneapolis, MN, USA) was used with a cell Berkovich point for the nanohardness and Young's modulus evaluation. Samples were individually placed on a computercontrolled X-Y table and were kept hydrated during the test. To ensure a precise transfer of the preprogrammed positions to the nanoindenter, an accurate calibration of the probe was performed on the standard fused quartz sample before the test's start. Five equally spaced $(10-\mu \mathrm{m})$ indentations were preprogrammed and performed on the dentin, hybrid layer, and adhesive layer, totaling 15 per specimen $(\mathrm{n}=3$, representing three specimens per group) with a load of $1000 \mu \mathrm{N}$ and a standard trapezoidal load function of 5-2-5 s. The nanohardness and Young's modulus of each area were computed as described elsewhere. ${ }^{16}$ Data were analyzed using one-way ANOVA (dentin treatment as factor; dentin, hybrid 
layer, and adhesive as levels) and Tukey's test for the SE and ER approaches.

\section{CA by Sessile Drop Method}

Human Teeth-Ten noncarious human third molars $(\mathrm{n}=5)$ were selected and stored in $0.5 \%$ chloramine/water $\left(4^{\circ} \mathrm{C}\right)$ immediately after extraction, then cleaned and stored in distilled water at $4^{\circ} \mathrm{C}$ to be used within three months after extraction. The teeth had their root and occlusal third removed using a diamond wafering blade (Buehler-Series 15HC Diamond, Buehler) on an automated sectioning device (Isomet 2000, Buehler) under running water. The exposed surface was ground with 600-grit $\mathrm{SiC}$ paper (Buehler-Met II, Buehler) under water irrigation. All surfaces of the dentin slices were carefully verified by stereomicroscopy for the absence of enamel/pulp tissue (Wild M5A, Wild, Heerbrugg, Switzerland).

Half of each tooth was treated with an NTAP brush for 10 or 30 seconds while a blade was used to separate and protect the other half, which was used as the untreated same-tooth control. This split of the sample was considered important since the standard deviation within dentin is large as it depends on different factors such as depth, age of the teeth, and number of tubules. ${ }^{17}$

Hydroxyapatite Plate-A commercially available hydroxyapatite plate $(10 \times 10 \times 2 \mathrm{~mm}$; APP100, Hoya, Tokyo, Japan) with a total area similar to an entire dentin flat slice dimension was prepared for CA evaluation. The purpose was to analyze an inorganic material present in dentin with and without the NTAP application. Comparing the results with dentin hydrophilicity should then estimate the plasma's influence on total and partial inorganic material. The blocks were also divided into two halves, and a blade protected one-half (control) from plasma treatment during the 10 - or 30 -second application.

CA Measurement: Three Repetitions in the Same Spot-Excess water on the dentin surfaces was gently blot dried with Kimwipes tissues (KimberlyClark, Roswell, GA, USA) before water CA measurement. Hydroxyapatite blocks were air-blown clean and kept dried before the experiment. The CA of distilled water was measured by the sessile drop technique with the use of a CAM200 goniometer (KSV Nima, Espoo, Finland), and the samples were kept in a $100 \%$ humidity chamber during measurement.

A drop of water (approximately $1.0 \mu \mathrm{L}$ ) was placed on one of the halves of the dentin surface $(n=3)$, and the image was immediately sent via the camera to the computer for analysis. Images were captured every five seconds at different water-deposition times (5-55 seconds).

The specimen images were analyzed by a computer program (Image $J$ software) with an angular dimension tool to measure the static CA. Right and left angles were measured to obtain a mean CA value. One drop of water was applied on each half of the sample surface: treated side and control side. After the first CA measurement, the sample was kept in position and blot-dried, and another drop of water was applied in the same spot following the same protocol. All measurements were done in triplicate.

CA Measurement: Immediate Analysis (Unrepeated)-The intriguing results of $\mathrm{CA}$ data after three repetitions led us to the decision to perform another test, recording the CA immediately after the plasma treatment, evaluating only one drop of water. In this way, it was possible to better assess the surface hydrophilicity change upon the treatment, without water intake interference. Therefore, 10 dentin and hydroxyapatite samples $(n=5)$ were prepared as described before, and the CA was individually evaluated in each half of the specimens.

\section{RESULTS}

\section{$\mu$ TBS}

All assumptions related to normal distribution and homoscedasticity were attended. No significant difference was found for the evaluation time factor $(p=0.22)$ in the ER approach, while the treatment factor $(p=0.02)$ and the interaction between the "evaluation time" and "treatment" $(p<0.0001)$ showed statistical differences. In the SE approach, the evaluation time factor was statically significant $(p<0.0001)$, while no difference was found for the treatment factor $(p=0.20)$ or the interaction between the "evaluation time" and "treatment" $(p=0.17)$. At the one-week evaluation, no differences among the treatments were found in either adhesive technique (Table 3). After two years of water storage in the ER evaluation, the NTAP 30-second group presented a higher mean $\mu$ TBS compared with the other groups. There were no pretest failures.

Cohesive failure within the composite resin was the most predominant failure pattern observed in all groups at both evaluation times (Figure 1). However, after aging, the incidence of adhesive and mixed failure increased, regardless of whether or not there was a plasma application. The factor "time" caused a 
Table 3: Microtensile Bond Strength (MPa) as a Function of Dentin Treatment and Storage Time for Each Adhesive Mode

\begin{tabular}{|llc|}
\hline \multicolumn{1}{|c}{ Treatment } & \multicolumn{2}{c|}{ Storage Time } \\
\cline { 2 - 3 } & \multicolumn{1}{c}{$\mathbf{1} \mathbf{~ w k}$} & $\mathbf{2} \mathbf{~ y}$ \\
\hline SE Control & $49.5(10.0) \mathrm{Aa}$ & $35.14(10.5) \mathrm{Ba}$ \\
\hline SE NTAP 10 s & $48.5(9.0) \mathrm{Aa}$ & $42.09(4.2) \mathrm{Ba}$ \\
\hline SE NTAP $30 \mathrm{~s}$ & $47.1(8.3) \mathrm{Aa}$ & $46.47(10.7) \mathrm{Ba}$ \\
\hline ER Control & $48.8(6.4) \mathrm{Aa}$ & $38.7(8.0) \mathrm{Ab}$ \\
\hline ER NTAP 10 s & $53.9(11.2) \mathrm{Aa}$ & $44.9(7.9) \mathrm{Ab}$ \\
\hline ER NTAP 30 s & 46.7 (7.0) Aa & $59.0(11.6) \mathrm{Aa}$ \\
\hline $\begin{array}{l}\text { a Values are given as mean (SD). Different uppercase letters indicate } \\
\text { significant differences ( } \mathrm{p} \leq 0.05) \text { between different storage times within the } \\
\text { same treatment, and different lowercase letters indicate significant } \\
\text { differences }(\mathrm{p} \leq 0.05) \text { between different treatments within the same aging } \\
\text { condition, for the same adhesive mode (self-etch [SE] or etch and rinse } \\
\text { [ER]). NTAP, nonthermal atmospheric plasma. }\end{array}$ \\
\hline
\end{tabular}

statistically significant difference in failure mode distribution $(p<0.0001)$. After 24 hours of storage, adhesive failures ranged between $7 \%$ and $15 \%$, increasing to between $20 \%$ and $46 \%$ after two years, whereas cohesive failures ranged from $65 \%$ to $85 \%$ at 24 hours, decreasing to $32 \%$ to $50 \%$ after aging. These higher ratios of adhesive failure after direct water exposure for a long time indicates degradation of the interfacial area, although the 30-second NTAP-treated groups did not show a significant statistical $\mu$ TBS reduction after two years of aging in ER mode.

\section{Nanohardness and Young's Modulus}

All assumptions related to normal distribution and homoscedasticity were attended. The mean values and standard deviation of nanohardness and Young's modulus are shown in Tables 4 and 5, respectively. The nanohardness of adhesive layer $(p=0.181)$ did not show significant differences among the groups. In the hybrid layer, the control groups showed lower nanohardness than their corresponding experimental groups. Lower dentin nanohardness was reported for NTAP 30-second groups.

The lowest dentin Young's modulus was recorded for the NTAP 30-second SE and ER groups, although it did not differ from the NTAP 10-second SE group. For both approaches (ER and SE), the groups treated with NTAP (10 and 30 seconds) showed a higher Young's modulus in the hybrid layer than their respective controls. There was no statistical difference among groups in the "adhesive layer" evaluation $(p=0.062)$.

\section{CA by Sessile Drop Method}

CA Measurement: Three Consecutives Repetitions in the Same Spot-The CAs of specimens treated with NTAP for 30 -seconds were significantly lower than those on the untreated dentin/hydroxyapatite surfaces (Figures 2 and 3). The dentin hydrophilicity slightly decreased after the 10 -second plasma treat-

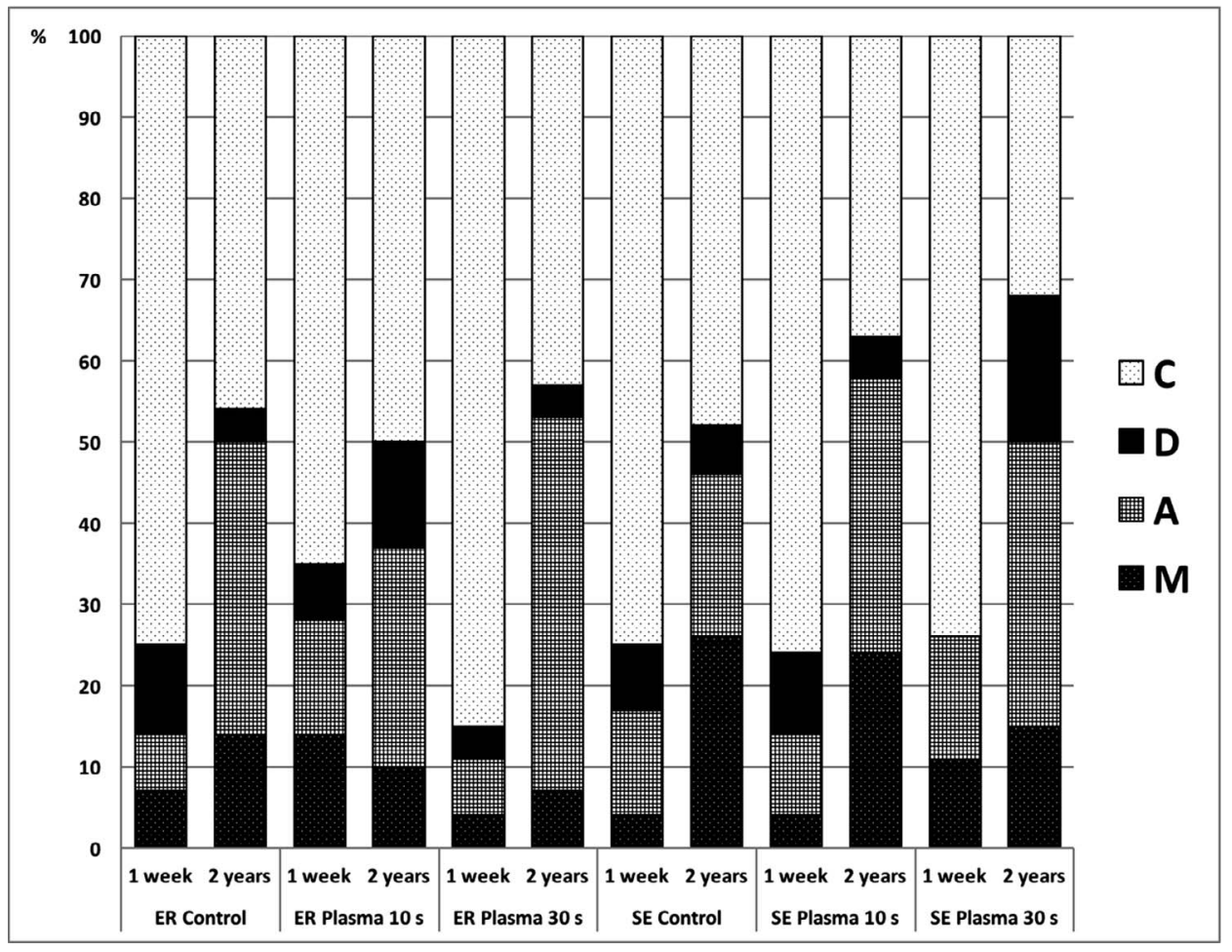

Figure 1. Distribution (\%) of failure modes of the groups tested after one week and two years of water storage. $C$, cohesive failure in composite resin; $D$, cohesive failure in dentin; $A$, adhesive failure; $M$, mixed failure of composite resin, adhesive, and dentin. 
Table 4: Nanohardness (GPa) Means (Confidence Interval) of the Dentin, Hybrid Layer, and Adhesive Layer ( $n=3$ ) of a Multimode Adhesive ${ }^{a}$

\begin{tabular}{|c|c|c|c|}
\hline Treatment & Dentin & Hybrid Layer & Adhesive Laye \\
\hline Control SE & $2.5(2.5-2.6) \mathbf{a}$ & $1.4(1.3-1.5) \mathbf{b}$ & $1.3(1.2-1.3) \mathbf{a}$ \\
\hline SE NTAP $10 \mathrm{~s}$ & $2.4(2.4-2.5) \mathrm{ab}$ & $1.6(1.5-1.7) \mathbf{a}$ & $1.3(1.3-1.3) \mathbf{a}$ \\
\hline Control ER & $2.5(2.4-2.5) \mathbf{a}$ & $1.2(1.2-1.3) \mathbf{b}$ & $1.3(1.3-1.3) \mathbf{a}$ \\
\hline ER NTAP $10 \mathrm{~s}$ & $2.6(2.5-2.7) \mathbf{a}$ & $1.5(1.5-1.6) \mathrm{a}$ & $1.3(1.3-1.4) \mathbf{a}$ \\
\hline ER NTAP $30 \mathrm{~s}$ & $2.0(2.0-2.1) \mathbf{b}$ & $1.4(1.4-1.5) \mathbf{a}$ & $1.3(1.3-1.4) \mathbf{a}$ \\
\hline
\end{tabular}

ment time, but the difference from the dentin control group was higher when the plasma was used for 30 seconds (Figure 2). The influence of the NTAP treatment in hydrophilicity was more evident in the hydroxyapatite samples (Figure 3), in which the CA deeply decreased even with just 10 seconds of the NTAP application. In CA measurement repetitions, the dentin/hydroxyapatite control groups remained close to the first CA means, while the NTAP 30second groups showed gradually higher CA results, both in the dentin and the hydroxyapatite.

CA Measurement: Immediate Analysis (Unrepeated)-Figure 4 shows the CA means of dentin and hydroxyapatite of the immediate plasma-treated and untreated (control) surfaces. In dentin, the discrepancy between the NTAP 30-second group and the control group was greater than the difference between the NTAP 10-second group and its control group. However, for the hydroxyapatite substrate, both treatment times produced a large difference in CA compared with the means in the control groups.

\section{DISCUSSION}

The benefits of applying plasma to dentin tissue are expected to act primarily on the longevity of adhesive restoration. Therefore, it is necessary to evaluate such effects as the function of the aging of the tooth-restoration interface. The first null hypothesis was partially accepted because the $\mu$ TBS of the plasma-treated dentin differed from the untreated group only in the evaluation of two years of aging when plasma was applied for 30 seconds in etched dentin.

Regarding the multimode adhesive evaluated in the present study, two years of water aging did not produce a statistically significant $\mu$ TBS reduction in values in the ER approach; however, the evaluation time factor was significant in the $\mathrm{SE}$ evaluation. Scotchbond Universal (SBU) adhesive's long-term effectiveness presents some conflicting results in the literature, ${ }^{6,18-21}$ although none of them evaluated such a long period of aging. The differences in the test design might explain such controversies; however, this multimode adhesive showed great durability under the present study conditions, especially in the ER approach, and no pretest failures were recorded for both times of evaluation.

On the contrary, unlike most investigations using plasma, ${ }^{2-6,11}$ in this study the NTAP treatment did not cause any difference in the immediate (oneweek) results of $\mu \mathrm{TBS}$. In the SE approach, no

\begin{tabular}{|c|c|c|c|}
\hline Treatment & Dentin & Hybrid Layer & Adhesive Layer \\
\hline NTAP $10 \mathrm{~s}$ & $35.5(33.0-38.0) \mathrm{ab}$ & $20.1(19.3-21.0) \mathbf{a}$ & $10.1(9.8-10.5) \mathbf{a}$ \\
\hline NTAP $30 \mathrm{~s}$ & $31.4(28.3-34.5) \mathbf{b}$ & $20.1(19.3-21.0) \mathbf{a}$ & $10.1(9.6-10.7) \mathbf{a}$ \\
\hline Control ER & $37.3(35.9-38.7) \mathbf{a}$ & $12.2(11.9-12.4) \mathbf{b}$ & $10.1(10.0-10.3) a$ \\
\hline NTAP $30 \mathrm{~s}$ & $33.4(31.1-35.6) \mathbf{b}$ & $14.7(14.0-15.3) \mathbf{a}$ & $10.7(10.0-11.4) \mathbf{a}$ \\
\hline
\end{tabular}




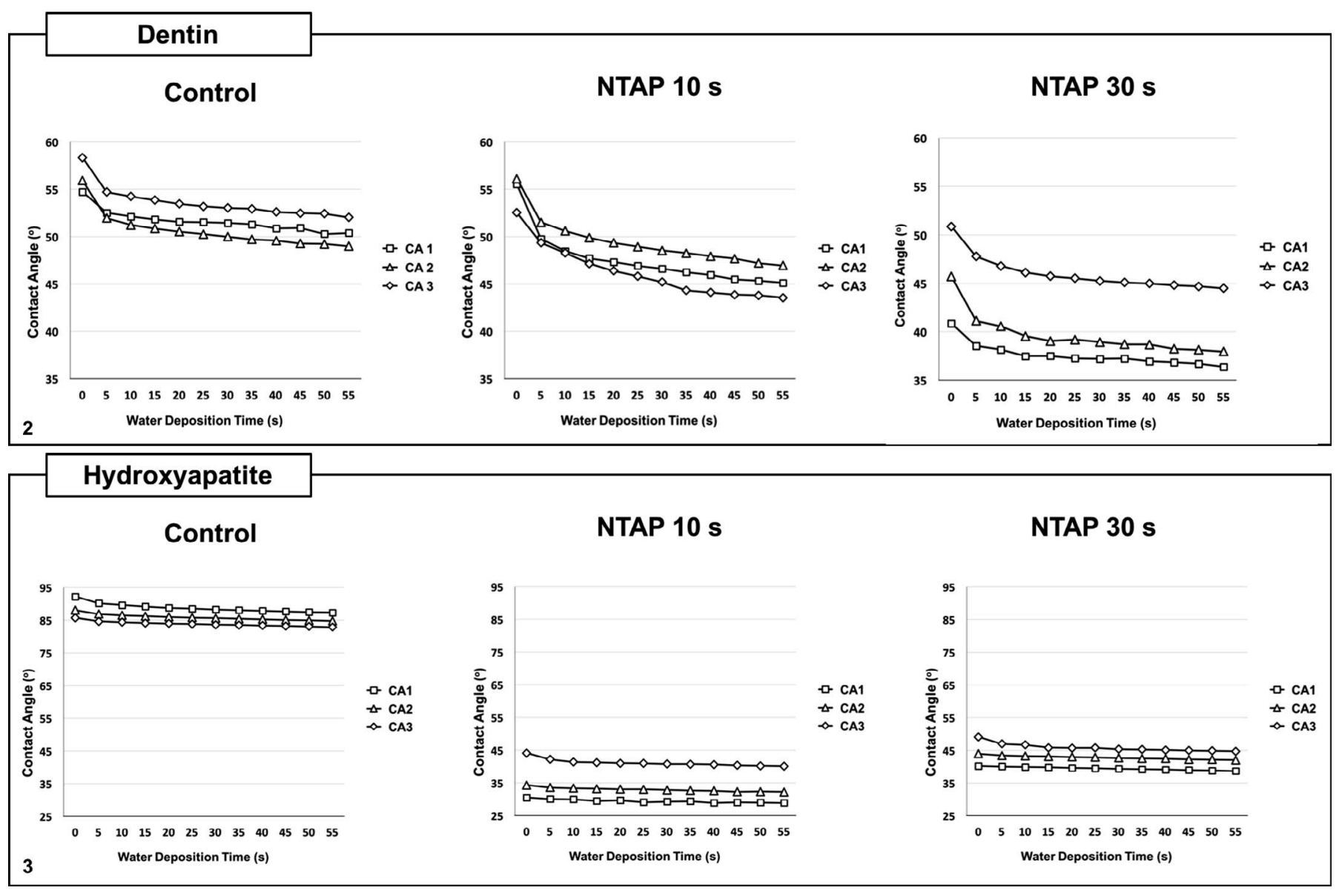

Figure 2. Means of water contact angle on dentin treated with NTAP for 10 or 30 seconds and compared with those on untreated dentin (control). $C A 1, C A 2, C A 3=$ first, second, and third contact angle measurements, respectively.

Figure 3. Means of water contact angle on hydroxyapatite treated with NTAP for 10 or 30 seconds and compared with those on untreated hydroxyapatite (control). CA1, CA2, CA3 = first, second, and third contact angle measurements, respectively.

statistical differences among treatments were observed after two years of water storage. Hirata and others ${ }^{6}$ also found the same performance in one-year aged plasma-treated groups associated with SBU in the SE approach.

In the present study, two years of water storage did not decrease dentin $\mu$ TBS for ER groups, and the two-year aged control group and NTAP 10-second group presented significantly lower $\mu$ TBS when compared with the NTAP 30-second group. There is today no clear consensus about the potential beneficial effects of NTAP, especially because most of the investigations did not evaluate long-term results. Different factors, such as specifications of the plasma device and the adhesive system used, makes it difficult to draw a direct comparison with other plasma studies, and this fact justifies the evaluation of a longer aging using the same parameters (adhesive system, direct water storage, NTAP application time/specifications).

However, studies from our research group using the SBU adhesive associated with the same plasma equipment (Surface Plasma Tool Model SAP) in standard specifications were recently published. SBU maintained its $\mu$ TBS strength to dentin after one year of direct water exposure and exposure to simulated pulpal pressure, although remarkable statistical differences between treatments were observed depending on the aging condition. ${ }^{22}$ A more recent method, mini-interfacial fracture toughness, showed no difference among plasma-treated and -untreated groups upon 6 months of aging, which could be explained by the short-time aging evaluation, once SBU also showed bonding stability in the present study even after two years of water aging. ${ }^{23}$

Although nanohardness and Young's modulus assessments were performed only one week after 


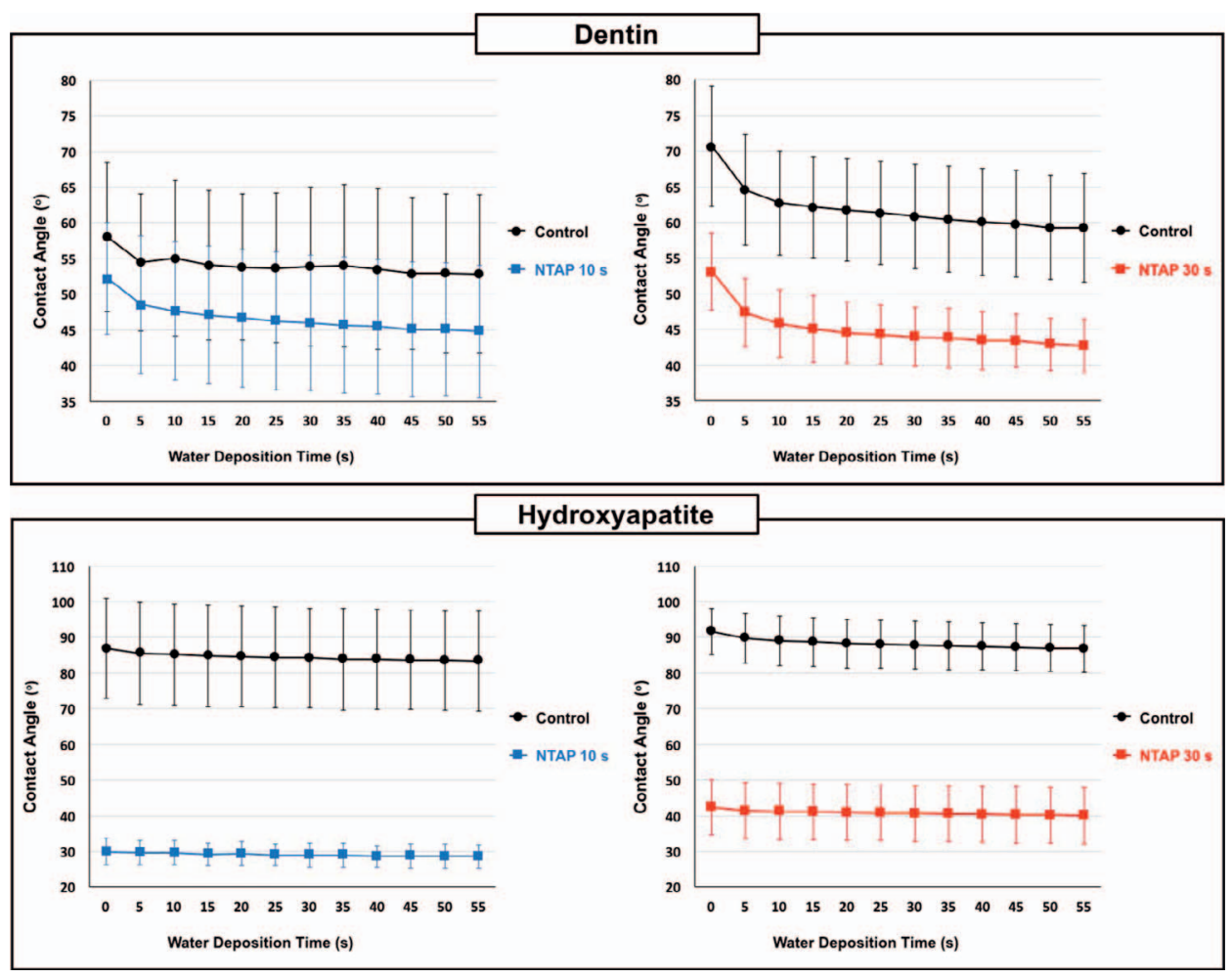

Figure 4. Means of water contact angle immediately after NTAP application for 10 or 30 seconds on the surfaces of dentin and hydroxyapatite surfaces compared with those on untreated (control) substrates.

applying NTAP, adhesives, and composite, they provided consistent and very rich information to explain the beneficial effects of NTAP on resin/ dentin-treated interfaces. When evaluating the hybrid layer, both application times of NTAP (10 seconds and 30 seconds) presented values of nanohardness and Young's modulus that were statistically higher than the respective untreated (control) groups. Thus, the second hypothesis was rejected because NTAP produced significant stiffening of the hybrid layer.

Another important observation was that no difference in nanohardness or Young's modulus was found in the adhesive layer (Tables 4 and 5). This result was also expected since the same adhesive resin was used in all the groups. However, the differences found in the hybrid layer raise the question about what caused the hardness increase in the specific area where the adhesive was in intimate contact with the plasma-modified surface.

The findings in the mechanical properties of the resin-dentin interface might be related to two possible alterations promoted by the NTAP application. One is the possible promotion of a higher crosslink density of the adhesive system, which may have originated from a greater number of chemical reactions, promoting the breaking of carbon chains. The plasma-treated dentin receives a jet of electrons, free radicals, and ions and therefore produces a more reactive surface, which can trigger more chemical reactions of the monomer components of the adhesive system. Chen and others ${ }^{10}$ applied a plasma brush in a model adhesive under different water/ HEMA mass ratios and demonstrated the plasma's effectiveness in inducing polymerization. Conversion values of the plasma-cured groups were higher than those of light-cured samples with the same mass ratio and water content.

Another possible explanation is that the adhesive system is able to interact strongly with partially demineralized dentin (in the SE mode) and fully demineralized dentin (in the ER technique), leaving less voids or water-filled spaces. An in situ zymography assay showed a progressive decline of enzymatic activity with increase of NTAP exposure time. ${ }^{22}$ Application of NTAP apparently inhibited the enzymatic activity in the ER specimens, especially in the hybrid layer area. The NTAP-treated dentin should demonstrate better infiltration and polymerization of resin monomers, corroborating the present findings of mechanical properties in the hybrid layer.

These theories might be confirmed by additional evaluations, such as transmission electron microscopy and Fourier transform infrared spectroscopy, in which the ultramorphology interaction between the 
adhesive and plasma-treated dentin can be observed as well as the degree of conversion of resin-based materials, respectively. In addition, long-term aging evaluation of nanohardness and Young's modulus of the resin-dentin interface's structures are incentivized. The correlation between $\mu$ TBS and nanomechanical properties of the resin-adhesive interface might be controversial. According to Freitas and others, this correlation was reported as inverse, suggesting that a lower Young's modulus for the adhesive layer offers more adequate resistance of the adhesive to elastic deformation under stress, although the difference was statistically significant only for the adhesive layer and the SBU adhesive was not evaluated. ${ }^{24}$ In the present study, the higher nanohardness and Young's modulus were related to the NTAP application effect on the hybrid layer, which also presented the highest $\mu$ TBS absolute means when applied for 30 seconds in dentin, indicating a positive correlation.

The supposition of a higher interaction of the adhesive system with the plasma-treated substrate was corroborated by the findings in the CA analysis by the sessile drop method. The NTAP treatment had a much stronger influence on the hydrophilicity of hydroxyapatite than of dentin. This finding indicates that the plasma effect is stronger on the inorganic content of the substrate. However, it is worth mentioning that the hydroxyapatite arrangement in the dentin substrate is much more complex, involving tubules with different distributions and diameters. ${ }^{16}$ In dentin, the NTAP application time of 10 seconds promoted a slightly lower CA mean than the control group, while the application for 30 seconds showed a more significant difference. Thus, the third hypothesis was also rejected.

In the triplicate test, every time that the application of the water droplet was repeated, the hydrophilicity decreased in the NTAP 30-second groups. Apparently, the presence of moisture on a surface after the NTAP application is likely to decrease the hydrophilicity potential. The highly reactive particles produced on the surface by NTAP can cross-link rapidly to form various chemical functional groups. ${ }^{10}$ The authors hypothesize that the droplets of water molecules may have acted as a contaminant, preventing the new water droplets from reacting with the plasma-modified surface, jeopardizing the wettability. This would also explain why the NTAP effect was more evident in the hydroxyapatite dry sample than in the partially wet dentin.

Therefore, it seems reasonable to counteract rehydration of the dentin after the NTAP application and to avoid contamination by water/saliva, letting only the adhesive system have direct contact with the plasma-treated substrate, allowing for a good infiltration. The NTAP application for longer time (30 seconds) produced higher hydrophilicity in dentin than the shorter time (10 second) in the first CA assessment and was more negatively affected in the third water droplet application. But it is worth noting that even after three water drop exposures, the NTAP treatment still maintained a higher hydrophilicity than the control groups in all water deposition times, especially on the hydroxyapatite substrate, which indicates the partial maintenance of hydrophilic properties.

Aiming to evaluate only the immediate effect of the NTAP treatment on surface hydrophilicity, the sessile drop CA method was performed again on new samples, but with only one measurement per spot. A larger number of samples were prepared to obtain a more representative mean since there were no repetitions. Once again, the NTAP effect on hydroxyapatite's hydrophilicity was much stronger than on the dentin's, even using the shorter treatment time (10 seconds), whose effect did not differ from the longer treatment time (30 seconds) in this particular substrate. In dentin, the application time for 30 seconds produced a more significant difference in the CA comparison with the control group.

Besides adhesion by contact, the presence of the 10-MDP monomer in the composition of the multimode adhesive tested in this study produces chemical reactions with calcium from hydroxyapatite, forming a hydrolytic stable dentin-resin interaction. ${ }^{25}$ Yoshida and others revealed Ca-salt formation and nanolayering within the hybrid layer; however, this additional bonding mechanism was not always equally consistent for SBU nor Clearfil SE Bond (Kuraray Noritake). ${ }^{26}$ According to those authors, more intense nanolayering was found in the areas with more demineralization. Because the dentin is a moist substrate, the preexisting and/or remaining water may result in more ionization of the acidic functional monomer once intense nanolayering is observed in the neighborhood of the dentin tubules.

Regarding the chemical surface characterization of NTAP-treated dentin surfaces, a study using Raman confocal microscopy showed no statistical difference in carbonate and collagen type I spectra in etched and unetched dentin groups after plasma application. ${ }^{22}$ They also reported no remarkable topographical alteration at the dentin surface when character- 
ized by atomic force microscopy. One could expect that the NTAP effect on surface hydrophilicity, especially on inorganic substrate, could also influence the chemical interaction potential of 10-MDP with hydroxyapatite. A dense nanolayered structure with a hydrophobic nature would help in protecting the resin-dentin interface against hydrolytic degradation effects.

\section{CONCLUSIONS}

Dentin NTAP treatment for 30 seconds influences the $\mu$ TBS of the multimode SBU adhesive in the ER approach after two years of water aging. This positive result might be correlated with the increase in nanohardness and Young's modulus of the hybrid layer and to the greater dentin hydrophilicity, two phenomena observed in short-time evaluation. Although some effects were observed using a plasma application for 10 seconds, the results suggest that 30 seconds is the most indicated treatment time.

\section{Acknowledgement}

The authors acknowledge the State of São Paulo Research Foundation (FAPESP) for financial support (2013/15952-7 and 2015/05939-9) of this study.

\section{Regulatory Statement}

This study was conducted in accordance with all the provisions of the local human subjects oversight committee guidelines and policies of the Commission for Medical Ethics of KU Leuven. The approval code for this study is: S57622.

\section{Conflict of Interest}

The authors of this article certify that they have no proprietary, financial, or other personal interest of any nature or kind in any product, service, and/or company that is presented in this article.

(Accepted 17 May 2018)

\section{REFERENCES}

1. Liu Y, Liu Q, Yu QS, \& Wang Y (2016) Nonthermal atmospheric plasmas in dental restoration Journal of Dental Research 95(5) 496-505.

2. Han GJ, Kim JH, Chung SN, Chun BH, Kim CK, Seo DG, Son HH, \& Cho BH (2014) Effects of non-thermal atmospheric pressure pulsed plasma on the adhesion and durability of resin composite to dentin European Journal of Oral Science 122(6) 417-423.

3. Dong X, Ritts AC, Staller C, Yu Q, Chen M, \& Wang Y (2013) Evaluation of plasma treatment effects on improving adhesive-dentin bonding by using the same tooth controls and varying cross-sectional surface areas European Journal of Oral Science 121(4) 355-362.

4. Ritts AC, Li H, Yu Q, Xu C, Yao X, Hong L, \& Wang Y (2010) Dentin surface treatment using a non-thermal argon plasma brush for interfacial bonding improvement in composite restoration European Journal of Oral Science 118(5) 510-516.

5. Dong X, Li H, Chen M, Wang Y, \& Yu Q (2015) Plasma treatment of dentin surfaces for improving self-etching adhesive/dentin interface bonding Clinical Plasma Medicine 3(1) 10-16.

6. Hirata R, Teixeira H, Ayres AP, Machado LS, Coelho PG, Thompson VP, \& Giannini M (2015) Long-term adhesion study of self-etching systems to plasma-treated dentin Journal of Adhesive Dentistry 17(3) 227-233.

7. Koban I, Duske K, Jablonowski L, Schröder K, Nebe B, Sietmann R, Weltmann KD, Hübner NO, Kramer A, \& Kocher T (2011) Atmospheric plasma enhances wettability and osteoblast spreading on dentin in vitro: proof-ofprinciple Plasma Processes and Polymers 8(10) 975-982.

8. Chen M, Zhang Y, Sky Driver M, Caruso AN, Yu Q, \& Wang Y (2013) Surface modification of several dental substrates by non-thermal, atmospheric plasma brush. Dental Materials 29(8) 871-880.

9. Lehmann A, Rueppell A, Schindler A, Zylla I-M, Seifert HJ, Nothdurft F, Hannig M, \& Rupf S (2013) Modification of enamel and dentin surfaces by non-thermal atmospheric plasma Plasma Processes and Polymers 10(3) 262-270.

10. Chen M, Zhang Y, Yao X, Li H, Yu Q, \& Wang Y (2012) Effect of a non-thermal, atmospheric-pressure, plasma brush on conversion of model self-etch adhesive formulations compared to conventional photo-polymerization Dental Materials 28(12) 1232-1239.

11. Zhang Y, Yu Q, \& Wang Y (2014) Non-thermal atmospheric plasmas in dental restoration: improved resin adhesive penetration Journal of Dentistry $\mathbf{4 2 ( 8 )}$ 1033-1042.

12. Van Meerbeek B, Willems G, Celis JP, Roos JR, Braem M, Lambrechts P, \& Vanherle G (1993) Assessment by nanoindentation of the hardness and elasticity of the resindentin bonding area Journal of Dental Research 72(10) 1434-1442.

13. Sadr A, Shimada Y, Lu H, \& Tagami J (2009) The viscoelastic behavior of dental adhesives: a nanoindentation study Dental Materials 25(1) 13-19.

14. Lopes B, Ayres AP, Lopes L, Negreiros W, \& Giannini M (2014) The effect of atmospheric plasma treatment of dental zirconia ceramics on the contact angle of water Applied Adhesion Science 2(1) 1-8.

15. Poitevin A, De Munck J, Van Landuyt K, Coutinho E, Peumans M, Lambrechts P, \& Van Meerbeek B (2007) Influence of three specimen fixation modes on the microtensile bond strength of adhesives to dentin Dental Materials Journal 26(5) 694-699.

16. Oliver W \& Pharr G (1992) An improved technique for determining hardness and elastic modulus using load and displacement-sensing indentation systems Journal of Materials Research 7(6) 1564-1583.

17. Tjäderhane L, Carrilho MR, Breschi L, Tay FR, \& Pashley DH (2009) Dentin basic structure and composition-an overview Endodontic Topics 2009 20(1) 3-29. 
18. Marchesi G, Frassetto A, Mazzoni A, Apolonio F, Diolosà M, Cadenaro M, Di Lenarda R, Pashley DH, Tay F, \& Breschi L (2014) Adhesive performance of a multi-mode adhesive system: 1-year in vitro study Journal of Dentistry 42(5) 603-612.

19. Rosa WL, Piva E, \& Silva AF (2015) Bond strength of universal adhesives: a systematic review and metaanalysis Journal of Dentistry 43(7) 765-776.

20. Muñoz MA, Luque-Martinez I, Malaquias P, Hass V, Reis $\mathrm{A}$, Campanha NH, \& Loguercio AD (2015) In vitro longevity of bonding properties of universal adhesives to dentin Operative Dentistry 40(3) 282-292.

21. Vermelho PM, Reis AF, Ambrosano GM, \& Giannini M (2017) Adhesion of multimode adhesives to enamel and dentin after one year of water storage Clinical Oral Investigations 21(5) 1707-1715.

22. Ayres AP, Bonvent JJ, Mogilevych B, Soares LES, Martin AA, Ambrosano GM, Nascimento FD, Van Meerbeek B, \& Giannini M (2017) Effect of non-thermal atmospheric plasma on the dentin-surface topography and composition and on the bond strength of a universal adhesive European Journal of Oral Sciences 126(1) 53-65.

23. Ayres APA, Pongprueksa P, De Munck J, Gré CP, Nascimento FD, Giannini M, \& Van Meerbeek B (2017) Mini-interfacial fracture toughness of a multimode adhesive bonded to plasma-treated dentin Journal of Adhesive Dentistry 19(5) 409-416.

24. Freitas PH, Giannini M, França R, Correr AB, CorrerSobrinho L, \& Consani S (2016) Correlation between bond strength and nanomechanical properties of adhesive interface Clinical Oral Investigations 21(4) 1055-1062.

25. Yoshida Y, Van Meerbeek B, Nakayama Y, Yoshioka M, Snauwaert J, Abe Y, Lambrechts P, Vanherle G, \& Okazaki M (2001) Adhesion to and decalcification of hydroxyapatite by carboxylic acids Journal of Dental Research 80(6) 1565-1569.

26. Yoshida Y, Nagakane K, Fukuda R, Nakayama Y, Okazaki M, Shintani H, Inoue S, Tagawa Y, Suzuki K, De Munck J, \& Van Meerbeek B (2004) Comparative study on adhesive performance of functional monomers Journal of Dental Research 83(6) 454-458. 